\title{
A CAUTIONARY VIEW OF THE USE OF CYCLIC PREFIX ON CDMA SYSTEMS
}

\author{
Cristiano Panazio * \\ EPUSP \\ LCS/PTC \\ Av. Prof. Luciano Gualberto, tr. 3, \\ Cid. Universitária, São Paulo SP, \\ Brazil, CEP 05508-900
}

\begin{abstract}
We call attention to the fact that, under certain conditions, the signal-to-noise ratio loss for a given $E_{b} / N_{o}$ value and the spectral efficiency degradation caused by cyclic prefix (CP) insertion may outweigh its benefits that is intersymbol interference elimination and better equalization when compared to an equivalent CDMA system without $\mathrm{CP}$ and with similar complexity frequency domain equalizer. In this paper, we analyze how such conditions (channel length and its frequency selectivity, $E_{b} / N_{o}$, number of active codes and equalizer length) affect the performance of both approaches in chip level equalization context.
\end{abstract}

\section{INTRODUCTION}

The proposal of the use of the cyclic prefix (CP) in OFDM (Orthogonal Frequency Division Multiplexing) was made by [1], where it was employed to make the received OFDM symbol look as it had been cyclically convoluted with the channel. Such property, which renders the correlation matrix circulant, allows to perfectly equalize a channel, in the absence of spectral nulls, with a finite number of coefficients, besides the elimination of intersymbol interference (ISI) and orthogonalization of the subcarriers. Using the FFT (Fast Fourier Transform), it is possible to achieve a very efficient implementation. These advantages and the success of OFDM systems in many areas have prompted the use of the CP in other modulation techniques as CDMA (Code Division Multiple Access) [2] and single-carrier systems [3]. Nonetheless, the addition of a CP or any other form of guard interval can be viewed as an addition of redundance, which incurs in spectral efficiency reduction and SNR (Signal-to-Noise Ratio) loss for a given $E_{b} / N_{o}$. Such drawbacks may not compensate its advantages, resulting in lower capacity and higher BER (Bit Error Rate). In this paper, we clarify when such situation might occur by analyzing system parameters as $E_{b} / N_{o}$, channel length and its frequency selectivity, system load (i.e., the number of active codes) and non-CP equalizer length, in the context of a downlink synchronous CDMA system using a MMSE (Minimum Mean Square Error) chip level equalizer.

\footnotetext{
*This work was funded by CAPES BEX 1869/01-9.
}

\author{
Maurice Bellanger \\ CNAM
}

Lab. Electronique et Communication

292, rue Saint-Martin, 75142, Paris,

France - Cedex 03

This paper is organized as follows. In section 2 we describe the system model. The MMSE chip level equalization is presented in section 3. Section 4 details the problems that may make the $\mathrm{CP}$ solution performs worse than the non $\mathrm{CP}$ approach. In section 5, we asses the impact of system parameters over the performance of both $\mathrm{CP}$ and non-CP approaches. Finally, in section 6 , the conclusion and perspectives are stated.

\section{SYSTEM MODEL}

The transmitted signal $x(m)$ is given by:

$$
x(m)=\sum_{l=0}^{N_{u}-1}\left\{a_{l}\left(\left\lfloor\frac{m}{N}\right\rfloor\right) q_{l}\left(\bmod (m)_{N}\right) s(m)\right\}
$$

where $l$ stands for the $l$-th user, $N_{u}$ is the total number of users, $a_{l}(m)$ is the $l$-th user's symbol, $q_{l}(m)$ is a WalshHadamard sequence, which elements can assume the values $\pm 1, \bmod (m)_{N}$ is the modulus of $m$ by $N$. The length of the spreading sequence (or spreading factor) is equal to $N$ and $s(m)$ is a random complex scrambling sequence that can assume the values $( \pm 1 \pm j) / \sqrt{2}$ with equal probability. For the sake of notation, we write $q_{l}\left(\bmod (m)_{N}\right) s(m)=c_{l}(m)$.

The received signal $u(m)$ is represented by:

$$
u(m)=\mathbf{h}^{T} \mathbf{x}(m)+n(m)
$$

where $\mathbf{h}=\left[\begin{array}{llll}h(0) & h(1) & \cdots & h(M-1)\end{array}\right]^{T}$ is the channel vector with $M$ coefficients, $\mathbf{x}(m)=[x(m) x(m-1) \cdots x(m-$ $M+1)]^{T}$ is the signal vector and $n(m)$ is an additive white Gaussian noise with variance $\sigma_{n}^{2}$. If we insert the CP, which is a copy of the last $N_{C P}$ chips of each spread symbol, and defining $N_{T}=N_{C P}+N$, the transmitted signal can be represented by:

$$
x_{C P}(m)=x\left(N\left\lfloor\frac{m}{N_{T}}\right\rfloor+\bmod \left(\bmod (m)_{N_{T}}-N_{C P}\right)_{N}\right)
$$

At the receiver, the first $N_{C P}$ samples of each symbol are discarded.

\section{MMSE CHIP LEVEL EQUALIZATION}

In the downlink, the orthogonality among the codes of a downlink synchronous CDMA system is broken by a multipath channel. One way to restitute the orthogonality is to 
use a decorrelator. However, we use a long code, created by a random scrambling sequence and thus the MAI (Multiple Access Interference) becomes not cyclostationary. Therefore, an adaptive implementation of the decorrelator is not possible. Nonetheless, another way to reduce the MAI consists in eliminating the multipaths, i.e., by equalizing the channel. This can be achieved by a chip level equalization before the despreading [4] for the non-CP system, using the following cost function based on the MMSE (Minimum Mean Square Error) criterion:

$J_{M M S E}(\mathbf{w})=\mathrm{E}\left\{\left|a_{l}\left(\left\lfloor\frac{m-d}{N}\right\rfloor\right) c_{l}(m-d)-\mathbf{w}^{H} \mathbf{u}(m)\right|^{2}\right\}$

where $\mathbf{w}=\left[\begin{array}{llll}w(0) & w(1) & \cdots & w(Q-1)\end{array}\right]^{T}$ is the equalizer coefficients vector and $Q$ is the equalizer length, $\mathbf{u}(m)=$ $[u(m) u(m-1) \cdots u(m-Q+1)]^{T}$ is the equalizer input vector, $d$ is the training sequence delay and $(\cdot)^{H}$ is the transpose and conjugate operation. The filter output $y(m)$ is given by:

$$
y(m)=\mathbf{w}^{H} \mathbf{u}(m)
$$

The despreading of the $p$-th symbol is accomplished by:

$$
\tilde{a}_{l}(p)=\sum_{m=(p N)}^{(p+1) N-1} y(m+d) c_{l}(m)
$$

The coefficients that minimizes (4) are given by:

$$
\mathbf{w}=\mathbf{R}^{-1} \mathbf{p}(d)=\left\{\sum_{l=0}^{N_{u}-1} \sigma_{a_{l}}^{2}\left(\mathcal{H H}^{H}\right)+\sigma_{n}^{2} \mathbf{I}\right\}^{-1} \sigma_{a_{l}}^{2} \mathcal{H}_{d}
$$

where $\mathbf{R}$ is the correlation matrix of the received signal, $\sigma_{a_{l}}^{2}$ is the power of the $l$-th user, $\mathcal{H}$ is the convolution matrix, $\sigma_{n}^{2}$ is the noise power and the cross-correlation vector $\mathbf{p}(d)$ is the $d$-th column of $\mathcal{H}$ multiplied by $\sigma_{a_{l}}^{2}$, represented here by $\sigma_{a_{l}}^{2} \mathcal{H}_{d}$. Assuming that all users have the same power, we can write (7) as:

$$
\mathbf{w}=\left\{N_{u}\left(\mathcal{H} \mathcal{H}^{H}+\eta \mathbf{I}\right)\right\}^{-1} \mathcal{H}_{d}
$$

where $\eta=\frac{\sigma_{n}^{2}}{N_{u} \sigma_{a}^{2}}$. If $\eta$ is small compared to the diagonal of $\mathcal{H} \mathcal{H}^{H}$, the equalizer will try to invert the channel. Otherwise, the equalizer will be closer to the channel matched filter. This behavior is more evident in the solution of the frequency domain equalizer for the $\mathrm{CP}$ technique, whose MMSE cost function is written as:

$$
J_{M M S E}(W(k))=\mathrm{E}\left\{\left|a_{l}(p) C_{l}(k, p)-W^{*}(k) U(k, p)\right|^{2}\right\}
$$

where $C_{l}(k, p)$ and $U(k, p)$ are the discrete Fourier transform (DFT) of $c_{l}(m)$ and $u(m)$ (with the CP already discarded at the reception) for the $k$-th beam and the $p$-th spread symbol, $W(k)$ is the one-tap equalizer coefficient of the $k$ th beam. Given that the DFT of a signal $y(m)$ is defined by $Y(k)=\sum_{m=0}^{N-1} y(m) \mathrm{e}^{-j 2 \pi k m / N}$ and that $c_{l}(m)$ has unitary variance, then $\mathrm{E}\left\{\left|C_{l}(k, p)\right|^{2}\right\}=N$ for any value of $k$. Hence, the solution that minimizes (9) is given by:

$$
W(k)=\frac{\sigma_{a}^{2} N H(k)}{N|H(k)|^{2} N_{u} \sigma_{a}^{2}+N \sigma_{n}^{2}}=\frac{H(k)}{N_{u}\left(|H(k)|^{2}+\eta_{C P}\right)}
$$

where $H(k)=D F T\{h(m)\}$ and $\eta_{C P}=\frac{\sigma_{n}^{2}}{N_{u} \sigma_{a}^{2}}$. From (10), when $\eta_{C P}$ is large compared to $|H(k)|^{2}$ for every value of $k$, the equalizer will be approximately the channel matched filter. If $\eta_{C P}$ is small, the equalizer will tend to the zero-forcing solution. This is the same behavior observed in (8). The filtering is obtained by:

$$
y(m, p)=\frac{1}{N} \sum_{k=0}^{N-1} W^{*}(k) U(k, p) \mathrm{e}^{j 2 \pi m k / N},
$$

with $p N \leq m<(p+1) N$ and the despreading is given by:

$$
\tilde{a}_{l}(p)=\sum_{m=p N}^{(p+1) N-1} y(m, p) c_{l}(m)
$$

In the simulations, we define the value of $\eta$ as being related to the non-CP technique. Given an $E_{b} / N_{o}$ value, the $\eta$ of the CP system $\left(\eta_{C P}\right)$ is higher due to the $S N R$ penalty incurred by the $\mathrm{CP}$ insertion. Making an optimistic assumption that the $\mathrm{CP}$ length $\left(N_{C P}\right)$ is equal to the channel length $M$, we have that $\eta_{C P}$ is related to $\eta$ by:

$$
\eta_{C P}=\frac{N+N_{C P}}{N} \eta=\frac{N+M}{N} \eta
$$

\section{PROBLEM STATEMENT}

The CP technique allows to implement an efficient frequency domain equalizer. Firstly, there is no training sequence delay to be optimized. For the non-CP solution, the optimum value of the training delay depends on the channel phase and the noise value. Usually, the more the channel is likely to be minimum phase, the smaller the training delay. Conversely, the more the channel is likely to be maximum phase, the larger is the training delay. For mixed phase channels, intermediary values tends to give better results. When the noise power increases, a behavior equivalent to that of a mixed phase channel can be observed. Another advantage is that if the CP has length equal to $M-1$ or it is larger than the channel, the equalizer behaves as an infinite equalizer even if it has a finite number of coefficients. Therefore, such characteristics allows the CP approach to achieve a better MSE in comparison to a non-CP solution and finite length equalizer for the same noise variance.

On the other hand, the addition of the CP incurs in a penalty in the SNR, given an $E_{b} / N_{o}$ value. On some channels, such disadvantage can be larger than the MSE increase due to not using the $\mathrm{CP}$ and will probably reflect on higher bit error rates. This is the main point that can make the CP approach worse than a non-CP solution. Additionally, if the BER of a system with CP is slightly lower than that of a non-CP system, it may not compensate the reduction of the spectral efficiency originated by the use of the CP.

In order to limit such impairments, it is recommended to keep the size of the CP lower than $20 \%$ of the transmitted block [5]. As a matter of fact, we could make the block 
as large as we want. However, the size of the block is constrained by the receiver complexity, latency and sensitivity to time-varying channels.

Usually, in favor of $\mathrm{CP}$, it is said that a large frequency domain equalizer with $\mathrm{CP}$ is much more efficient $\left(\mathcal{O}\left(N \log _{2} N\right), N\right.$ is the FFT length) than a temporal domain equalizer $\left(\mathcal{O}\left(N^{2}\right)\right.$, where $N$ is the equalizer length) for non$\mathrm{CP}$ systems. This statement is true. However, the frequency domain implementation of the equalizer for the non-CP systems using overlap-add or overlap-save [6] can reduce its complexity to $\mathcal{O}\left(2 \operatorname{Nlog}_{2} 2 \mathrm{~N}\right)$. Hence, for the same number of coefficients, the non-CP technique is approximately 2 times more complex than the $\mathrm{CP}$ technique. Thus, the advantage of enlarging the block to reduce the impact of the $\mathrm{CP}$ insertion is also an advantage for the non-CP system, since the ratio of their complexities will not change. By the way, if $N \rightarrow \infty$ both equalizers presents the same performance. Therefore, the larger the equalizer in relation to the $\mathrm{CP}$, and equivalently, the larger the equalizer of a non-CP system, the smaller is the difference between them.

In the next section, we shall quantify this trade-off by taking into account the system parameters as channel length and its frequency selectivity, $E_{b} / N_{o}$, number of active codes, equalizer length of the non-CP approach and spectrum efficiency.

\section{CP VERSUS NON-CP APPROACH}

\subsection{Influence of the channel and equalizer lengths}

As a matter of fact, we could pose the question of which is the relation of performances between CDMA systems that uses the $\mathrm{CP}$ approach or not. For the non-CP solution, the performance of a finite linear equalizer will be lower when the channel presents high frequency selectivity (e.g., $H(z)=1+z^{-1}$ ) or when it is about the size of the equalizer or when the value of $\eta$ is small. In all cases, more coefficients are needed to achieve better equalization. Hence, a small equalizer may have significant higher MSE than a CP technique, which allows to achieve the performance of an infinite length non-CP equalizer. However, as we discussed in section 4 , for a given $E_{b} / N_{o}$ value, the CP insertion decreases the SNR that raises the MSE. This may translate into higher BER. Thus, in order to compare the MSE of CP and non-CP techniques, we have chosen the following channel:

$$
H(z)=\frac{\alpha+\rho \beta z^{-\tau}}{\sqrt{1+\rho^{2}}}
$$

where $\alpha$ and $\beta$ are complex random variables with normalized variance, $\rho$ and $\tau$ are the gain and the delay of the second path respectively. In the two path channel, the delayed copies of the transmitted signal (ISI) are concentrated on the most (and only) delayed path, which should maximize the noxious effects of the ISI. Therefore, this channel is an optimistic choice for the CP approach, since it enables the better use of one of the $\mathrm{CP}$ advantages that is the elimination of ISI. By fixing $\eta$, it is possible to trace the MSE surfaces as a function of $\rho$ and $\tau$. Since we do not know if the channel will have minimum or maximum phase, it was chosen a conservative value for the training delay used in the non-CP approach that is half of the equalizer length. The spreading factor $N$ is equal to 64 , which already provides a small granularity for the analysis and thus we can extrapolate the results for higher $N$ values. For all techniques, the optimal coefficients were analytically obtained by (8) and (10). The MSE for each $\rho$ and $\tau$ is obtained by averaging over $2 \times 10^{4}$ channel realizations. The CP length is equal to $\tau$, which is an optimistic assumption for such technique. The MSE for the non-CP approach is written as:

$$
M S E_{\text {non-CP }}(\mathbf{w})=\sigma_{x}^{2}-\sigma_{x}^{2} \mathbf{w}^{H} \boldsymbol{H}_{d}
$$

and for the $\mathrm{CP}$ approach,

$$
M S E_{C P}(W)=N \sigma_{x}^{2}-\sigma_{x}^{2} \sum_{k=0}^{N-1} W^{*}(k) H(k)
$$

or in time domain

$$
M S E_{C P}(\mathbf{w})=\sigma_{x}^{2}-\sigma_{x}^{2} \mathbf{w}^{H} \mathbf{h}
$$

where $w(m)=\frac{1}{N} \sum_{k=0}^{N-1} W(k) \mathrm{e}^{j 2 \pi \frac{m k}{N}}$ and $0 \leq m<N$.

The higher the power of the second path, the largest the chance that the channel will get more selective. From the MMSE solution, the more selective the channel, the lower is the performance of a finite non-CP equalizer. In the same way, the longer the channel, the harder to equalize it with a finite non-CP equalizer. From figure 1, for small values of $\rho$ and $\tau$, the $\mathrm{CP}$ solution is less effective than the non-CP approach. However, we can clearly see that when one or both values grow, the $\mathrm{CP}$ approach becomes more efficient and provides better MSE. It can be expected that the region where the non-CP approach is better than the $\mathrm{CP}$ solution will shrink if we reduce the non-CP equalizer length $(Q<$ $N)$. Such behavior is shown in figure 2, where we reduced the number of non-CP equalizer length to 32 . On the other hand, if we can make an equalizer with more coefficients than the number of coefficients used in the CP approach, we could extend the region where the non-CP solution is more advantageous at the expense of higher complexity.

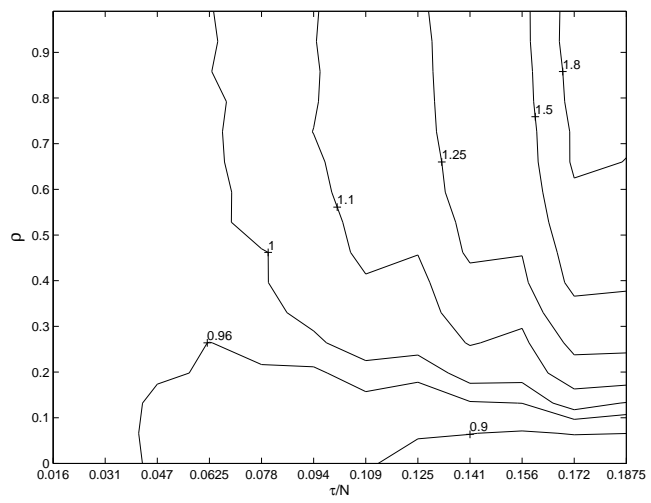

Fig. 1. $\frac{M S E_{n o n-C P}}{M S E_{C P}}$ for $\eta=0.0158, N=64$ and non-CP equalizer length $Q=64$. 


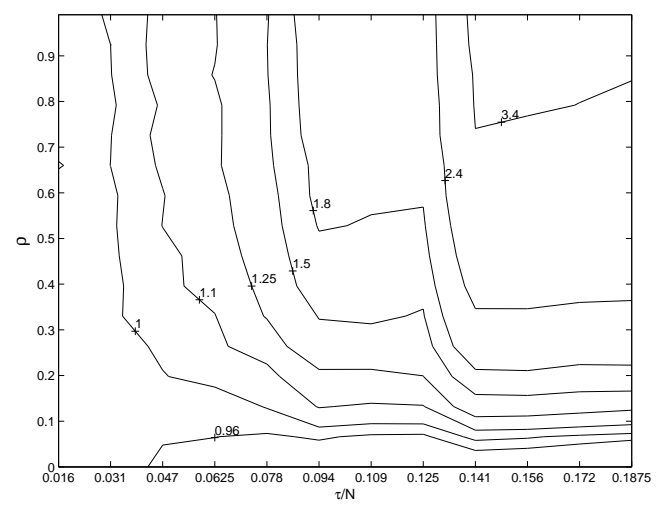

Fig. 2. $\frac{M S E_{n o n-C P}}{M S E_{C P}}$ for $\eta=0.0158, N=64$ and non-CP equalizer length $Q=32$.

\begin{tabular}{|l|l|l|l|l|l|}
\hline Path & 0 & 1 & 2 & 3 & 4 \\
\hline Power $[\mathrm{dB}]$ & 0 & -1 & -3 & -6 & -9 \\
\hline Delay $\left[T_{c}\right]$ & 0 & 2 & 6 & 9 & 12 \\
\hline
\end{tabular}

Table 1. 5-path channel used to show the influence of $\eta$ parameter.

The next step was to (indirectly) analyze a Rayleigh channel with a fixed length and power profile the performances of techniques in terms of BER, the number of active codes and $\mathrm{E}_{\mathrm{b}} / \mathrm{N}_{\mathrm{o}}$.

\subsection{Performance as a function of $E_{b} / N_{o}$ and number of active codes}

We have chosen the multipath channel described in table 1 and QPSK (Quadrature Phase-Shift Keying) modulation in all following simulations. The chip period $\left(T_{c}\right)$ is equal to $26,04 \mu$ s and the spreading factor $(N)$ is equal to 64 . The Doppler frequency $\left(f_{d}\right)$ was set to $111 \mathrm{~Hz}$. Again, the optimal coefficients were analytically obtained by (8) and (10).

From figure 3 , for small values of $E_{b} / N_{o}$ and 64 active users, the non-CP solution performs better than the CP approach. However, when $E_{b} / N_{o}$ increases, the performance gap between these techniques is reduced and eventually the $\mathrm{CP}$ solution becomes better while the non-CP approach converges to what appear to be an error floor. It is worth noting that the performance difference inverts only for a raw BER around $3 \times 10^{-4}$, which is already a small value, considering that forward error correction will be used. On the other hand, for the case with 16 active codes, where the equalizer is less stressed ( $\eta$ is larger), the non-CP approach is always better than the CP solution for the simulated $E_{b} / N_{o}$ range. Such behavior comes from the fact that for a higher $E_{b} / N_{o}$ value or/and when the system is near full load (i.e., with almost all codes being used), the value of $\eta$ decreases. Hence, the solutions presented in equations (8) and (10) tend to approach the zero-forcing solution where the equalizer will try to fully invert the channel. In this case, the larger the equalizer, the better its performance. Thence, the equalizer used in $\mathrm{CP}$ approach, which behaves as an infinite length equalizer, has a performance advantage over the non-CP solution,

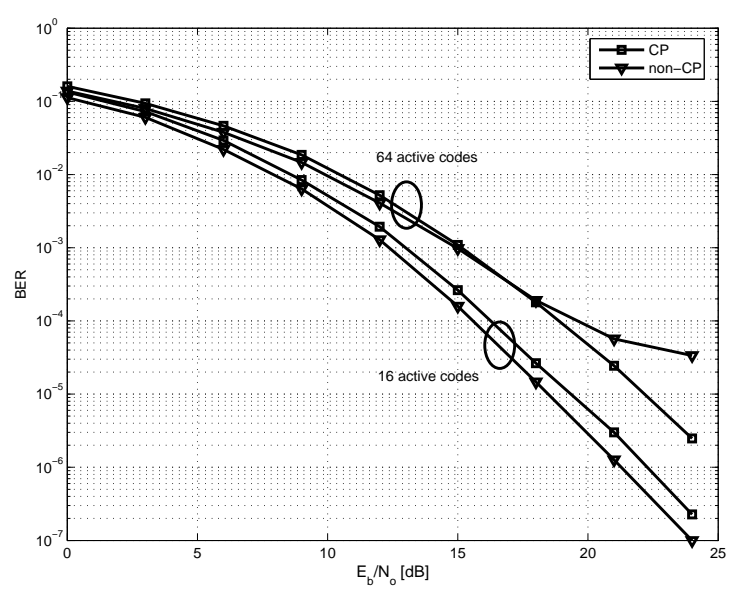

Fig. 3. Raw BER for the channel in table $1, N=64, T_{c}=$ $20,04 \mu$ s and $f_{d}=111 \mathrm{~Hz}$.

in particular when the channel is hard to equalize, i.e., when the channel is highly selective. Such advantage will eventually compensate the $E_{b} / N_{o}$ degradation caused by the CP insertion.

We also compared coded systems in figure 4 . The code used in the simulations is a half-rate convolutional code with polynomial generators given by $[5375]_{\text {octal }}$. We have used a random interleaver and all active codes were used to send data to only one user.

The use of forward error correction helps the non-CP technique by raising the value of $\eta$ due to the redundance insertion. In this case, $\eta=\frac{\sigma_{n}^{2}}{N_{u} \sigma_{a}^{2}}$ becomes $\eta=\frac{\sigma_{n}^{2}}{R_{F E C} N_{u} \sigma_{a}^{2}}$, where $R_{F E C} \leq 1$ is the coding rate. If powerful codes are used in conjunction with low order modulations, which let us work in low $E_{b} / N_{o}$ ranges, it is very likely that the non-CP technique will perform better than the $\mathrm{CP}$ solution. Also, one may argue that the last paths (3 and 4, table 1) are inexpressive in comparison with the others paths. Thus, it may be worthwhile to reduce the CP length. Actually, the equalizer for cyclic convolution is very sensitive to the wrap-around effect, which may lead to BER degradation that is larger than the SNR gain due to the reduction of the $\mathrm{CP}$ length. Such problem is also illustrated in figure 4. For a target $\mathrm{BER}=10^{-5}$, the non-CP solution provides $0.8 \mathrm{~dB}$ gain over the full length $\mathrm{CP}$. For other $\mathrm{CP}$ lengths such difference increases (e.g., $1.6 \mathrm{~dB}$ for $N_{C P}=6$ ).

The BER curves do not take into account the impacts on spectral efficiency. In order to consider both BER and spectral efficiency, we make use of the concept of Goodput. It measures the actual data successfully transmitted and is defined as:

$$
\text { Goodput } \triangleq R_{\text {raw }} R_{F E C} R_{C P}(1-P E R)
$$

where $R_{\text {raw }}$ is the raw physical throughput, that takes into account the constellation size and symbol period, $R_{F E C}$ is the coding rate, $R_{C P}$ represents the $\mathrm{CP}$ overhead and PER is the packet error rate, which is a function of the BER. We can make both CP and non-CP systems have the same 


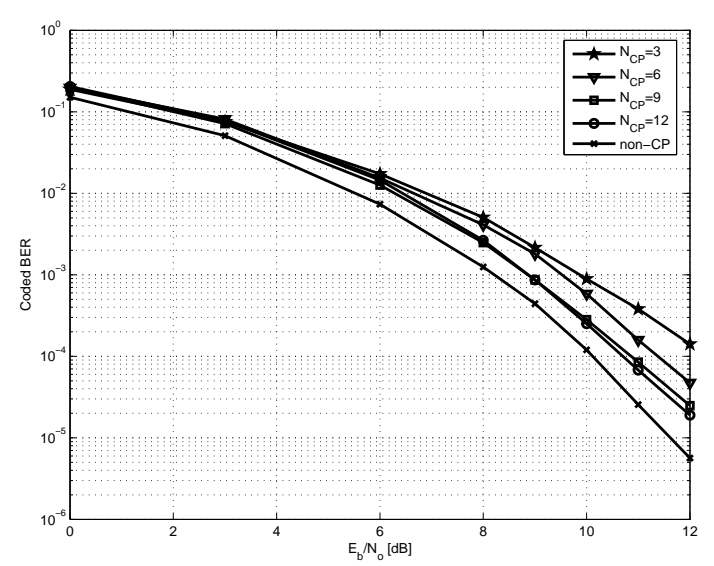

Fig. 4. Coded BER for the channel in table $1, N=64$, $T_{c}=20,04 \mu \mathrm{s}$ and $f_{d}=111 \mathrm{~Hz}$.

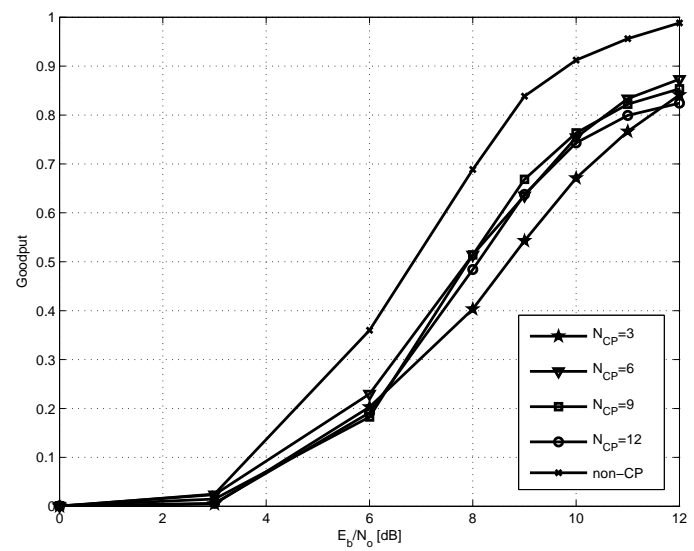

Fig. 5. Goodput for the channel in table $1, N=64, T_{c}=$ $20,04 \mu$ s and $f_{d}=111 \mathrm{~Hz}$.

$R_{\text {raw }}$. The coding rate $R_{F E C}$ is equal to 0.5 . For the sake of comparison, let us make $R_{\text {raw }} R_{F E C}=1$ without loss of generality. The CP overhead is $R_{C P}=\frac{N}{N+N_{C P}}=\frac{\eta}{\eta_{C P}}$. The PER was calculated for a packet size of 13120 data bits.

The results presented in figure 5 shows that the non$\mathrm{CP}$ approach allows not only better Goodput for a given $E_{b} / N_{o}$, but it also allows to achieve a higher capacity in this channel. It is worth noting that the augmentation of $R_{C P}$ by the CP size reduction counterbalances the higher PER (and higher BER) and allows to achieve the same or slightly higher Goodput values on the analyzed interval. However, this is true till a certain point. For example, when $N_{C P}=$ 3 , the Goodput is considerably decreased for the analyzed $E_{b} / N_{o}$ range.

\section{CONCLUSIONS}

We call attention to the fact that for chip-level equalization of CDMA systems depending on the channel length and frequency selectivity, $E_{b} / N_{o}$, number of active users and equalizer length, the use of cyclic prefix (CP)-CDMA may result in performance loss when compared to similar complexity frequency domain implementation without CP. By comparing the MSE of both techniques, we were able to determine that $\mathrm{CP}$ approach is more efficient when the channel equalization is harder to obtain, i.e., a larger equalizer is required. When comparing raw BER, it is shown that in low $E_{b} / N_{o}$ regions or when the system load is not at its maximum, the non-CP approach tends to perform better. This advantage arises from the fact that in these situations the equalizer tends to the channel matched filter, which requires less coefficients than the situation where the channel needs to be inverted. The same conclusion has shown to be valid on coded systems. In terms of Goodput, the non-CP technique can provide higher rates. We can conclude that the $\mathrm{CP}$ approach tends to be more effective for highly selective channels, high order modulations that needs large values of $E_{b} / N_{o}$ and high rate codes.

It is worth noting that the same analysis is also valid for multicarrier (MC)-CDMA, since it can be viewed as a dual of the direct sequence (DS) version studied here [7].

How to map the two path analysis to any other channel would be very interesting, since such general result can be used as a guideline for system design. A natural and direct extension of this work is to provide a comparison of single-carrier (SC) and CP-SC modulations and for other equalization techniques, in the same basis that was done in this paper.

\section{REFERENCES}

[1] A. Peled and A. Ruiz, "Frequency domain data transmission using reduced computational complexity algorithms," in Proc. of the IEEE ICASSP, Denver, USA, April 1980.

[2] F. Adachi, T. Sao, and T. Itagaki, "Performance of multicode DS-CDMA using frequency domain equalisation in frequency selective fading channel," IEE Electronics Letters, vol. 39, no. 2, pp. 239-241, January 2003.

[3] D. Falconer, S. L. Ariyavisitakul, A. Benjamin-Seeyar, and B. Edison, "Frequency Domain Equalization for Single-Carrier Broadband Wireless Systems," IEEE Comm. Magazine, pp. 58-66, Abril 2002.

[4] K. Hooli, M. Latva-aho, and M. Juntti, "Multiple access interference suppression with linear chip equalizers in WCDMA downlink receivers," in Proc. IEEE Global Comm. Conf., (Globecom), Rio de Janeiro, Brazil, December 1999, pp. 467-471.

[5] H. Sari, G. Karam, and I. Jeanclaude, "Transmission techniques for digital terrestrial TV broadcasting," IEEE Comm. Magazine, vol. 33, 1995.

[6] J. J. Shynk, "Frequency domain and multirate adaptive filtering," IEEE Signal Proc. Magazine, pp. 14-37, January 1992.

[7] C. Panazio, Étude fréquentiel de l'étalement de spectre et impact sur la conception d'un récepteur de radiocommunication universel, Ph.D. thesis, CNAM, May 2005. 